\title{
Creating Summaries from User Videos
}

Michael Gygli ${ }^{1,2}$, Helmut Grabner ${ }^{1,2}$, Hayko Riemenschneider ${ }^{1}$, and Luc Van $\mathrm{Gool}^{1,3}$

1 Computer Vision Laboratory, ETH Zurich, Switzerland

2 upicto $\mathrm{GmbH}$, Zurich, Switzerland

${ }^{3}$ K.U. Leuven, Belgium

\{gygli,grabner, hayko, vangool\}@vision.ee.ethz.ch

\begin{abstract}
This paper proposes a novel approach and a new benchmark for video summarization. Thereby we focus on user videos, which are raw videos containing a set of interesting events. Our method starts by segmenting the video by using a novel "superframe" segmentation, tailored to raw videos. Then, we estimate visual interestingness per superframe using a set of low-, mid- and high-level features. Based on this scoring, we select an optimal subset of superframes to create an informative and interesting summary. The introduced benchmark comes with multiple human created summaries, which were acquired in a controlled psychological experiment. This data paves the way to evaluate summarization methods objectively and to get new insights in video summarization. When evaluating our method, we find that it generates high-quality results, comparable to manual, human-created summaries.
\end{abstract}

Keywords: Video analysis, video summarization, temporal segmentation

\section{Introduction}

With the omnipresence of mobile phones and other consumer oriented camera devices, more and more video data is captured and stored. To find and access relevant videos then quickly becomes a challenge. Moreover, the easier and cheaper video acquisition becomes, the more casual and sloppy the average quality typically gets. The automated production of good video summaries, that capture the important information and are nice to watch, can mitigate both issues.

One way of coping with the search challenge is visual indexing, where keyframes are selected such that they best summarize the video $[28,5,1,13,18,15,16]$. Keyframes are typically extracted using change detection [5] or clustering based on low-level features [1] or objects [18]. Others resort to web priors to find important frames within a video $[15,16,20]$. While keyframes are a helpful way of indexing videos, they are limited in that all motion information is lost. That limits their use for certain retrieval tasks (e.g. when looking for a nice panning shot from the top of the Eiffel tower), but renders them even less useful for improving the viewing experience. Therefore, video skimming, i.e. replacing the video by a shorter compilation of its fragments, seems better suited for such goals. This is 


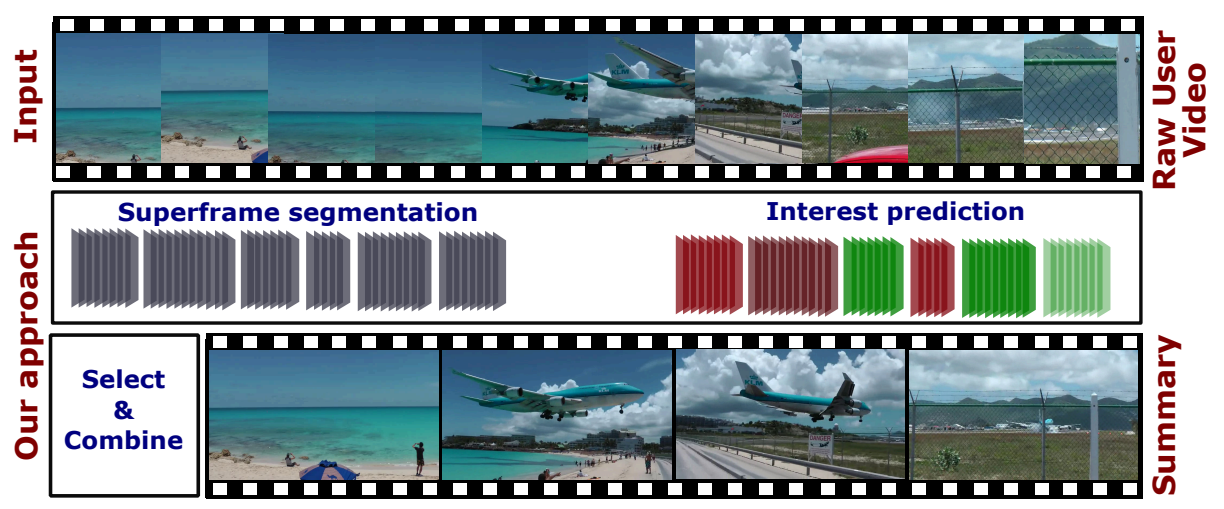

Fig. 1: Overview of our approach. First, we segment the video into superframes (cuttable segments). Then, we predict the interestingness (using low-level features and face/person, landmark detectors and motion features) for each superframe. From these, we select an optimal subset.

however a challenging task, especially for user videos, as they are unstructured, range over a wide variety of content and what is important often depends on a semantic interpretation.

Early work on the summarization of edited videos, such as tv news, is by Smith and Kanade [26], who detect camera motion, shot boundaries and faces, among other things, to create an automatic summary. Liu et al. [21] proposed a framework to summarize BBC rushes based on low-level cues, that clusters frames and uses image saliency and the visual differences between frames to score them. Ejaz et al. [5] follow a very similar approach to score frames, but use a non-linear fusion scheme. Several approaches target video summarization on a semantic level $[22,19,18,9]$, but as the reliable detection of high-level information, such as objects, is still an open research problem, many of them take user annotations (e.g. object bounding boxes) as input $[19,22,9]$.

Probably the most related work to ours are the recent works done at UT Austin [18,22]. They summarize long, raw, egocentric videos into keyframes [18] or skims [22], using an object-centered approach. In order to find important objects in a video, [18] uses object segmentations and a set of object-centered features, while [22] analyzes how objects link a set of events in a story. The usefulness of their approach was confirmed in a human study, were subjects were asked to compare the proposed summaries to several baselines.

In contrast to $[18,22]$ we introduce a more generic algorithm that summarizes any type of video (static, egocentric or moving), while taking into account cinematographic rules. Thereby we focus on user videos, which we define as unedited video data, that was taken with a purpose. Such video data often contains a set of interesting events, but is raw and therefore often long, redundant and contains parts of bad quality. Our goal is therefore different from [18, 22], who summarize video from wearable cameras, which often run for hours. Since user videos con- 
tain a wide range of content, solely relying on object-centric features, as in [18], is insufficient in our case. Therefore we propose new features better suited for the task of summarizing user videos.

Rather than manually evaluating the produced summaries, as [18,22], we introduce a new benchmark of user videos ranging over different categories. We evaluate our method using multiple 'ground truth' summaries per video, which we acquired in a study in collaboration with perception psychologists. This data allows to assess the performance of any summarization algorithm in a fast and repeatable manner.

We make the following contributions:

i) Superframes. A novel approach for motion-based video over-segmentation using insights from editing theory (Sec. 3). As these superfames have their boundaries aligned with positions appropriate for a cut, they create an aesthetic summary when combined.

ii) Summarization of user videos. A new method to estimate the interestingness of superframes and selecting a summary from them using a 0/1knapsack optimization. With this formulation the interestingness contained in the final summary is maximized, while remaining within a desired time budget (Sec. 5).

iii) The SumMe benchmark. A new, publicly available dataset of user videos that allows for an objective and repeatable evaluation of video summarization methods. To the best of our knowledge, it is the first that is annotated with human scores for video segments rather than keyframes and that allows for an automatic evaluation of different methods $^{4}$ (Sec. 6).

\section{Overview}

An overview of our approach to create an automatic summary is shown in Fig. 1. We start by over-segmenting a video $\mathcal{V}$ into superframes $\mathcal{S}$ (Sec. 3). Superframes are sets of consecutive frames where start and end are aligned with positions of a video that are appropriate for a cut. Therefore, an arbitrary order-preserving subset can be selected from them to create an automatic summary. Inspired by a recent work on human interest in images [11], we then predict an interestingness score $I\left(S_{j}\right)$ for each superframe (Sec. 4). For this purpose, we use a combination of low-level image features, motion features, as well as face/person and landmark detectors. Finally, we select an optimal subset of $\mathcal{S}$, such that the interestingness in the final summary is maximized (Sec. 5).

\section{Superframe Segmentation}

Traditional video summarization methods are focused on edited videos, such as news stories, sport broadcasts or movies. As these videos are edited, they consist

${ }^{4}$ Dataset and evaluation code are available on: www.vision.ee.ethz.ch/ gyglim/vsum/ 

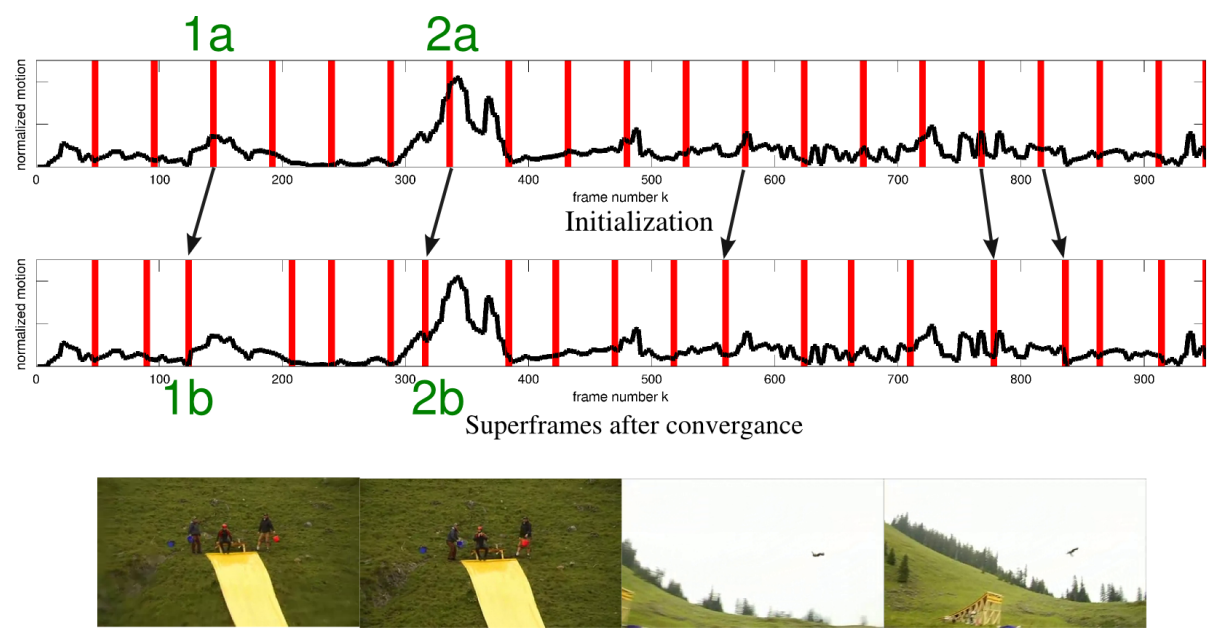

1a In zoom out $\quad 1 b$ before zoom out 2a During fast pan $2 b$ Before fast pan

Fig. 2: Superframe segmentation illustration. Superframes are initialized with a uniform length and then iteratively adapted to the motion. This leads to segments which have boundaries with less motion and often enclose a distinct event.

of a set of short shots. In order to segment such a video, it is sufficient to use shot detection, e.g. based on changes in the color histogram [26]. As we focus on largely unedited user videos, which often only contain one single shot, such an approach cannot be used in our case. This problem was also targeted earlier by [22], who proposed to classify frames from egocentric videos into static, in transit or head movement, in order to segment a video into shots. This method is however only applicable for egocentric videos and leads to shots of about 15 seconds, which is much longer than what people typically choose to summarize a video (see Fig. 3). A more general option would be to cut the video into segments of fixed length, but such arbitrarily cut shots would not correspond to logical units of the videos. In addition, this would lead to disrupting cuts, as humans are irritated by abrupt motion changes caused by cuts [24, p. 161].

As a remedy, editing guidelines propose to cut when there is no motion (or else, the motion speed and direction of two neighboring shots is matched) [24, p. 158-161]. We design a subshot segmentation that incorporates this idea. We term these segments superframes, in analogy to superpixels, and propose an approach inspired by recent work in image segmentation [2].

We define an energy function $E\left(\mathcal{S}_{j}\right)$ that is a measure of quality of a superframe $\mathcal{S}_{j}$ as

$$
E\left(\mathcal{S}_{j}\right)=\frac{1}{1+\gamma C_{c u t}\left(\mathcal{S}_{j}\right)} \cdot P_{l}\left(\left|\mathcal{S}_{j}\right|\right),
$$

where $C_{c u t}$ is the cut cost and $P_{l}$ is a length prior for the superframes. $|\cdot|$ denotes the length of a superframe. The parameter $\gamma$ controls the influence between the cut cost and the length prior, where a lower $\gamma$ leads to more uniform superframes. 
The cut cost is defined as

$$
C_{\text {cut }}\left(\mathcal{S}_{j}\right)=m_{\text {in }}\left(\mathcal{S}_{j}\right)+m_{\text {out }}\left(\mathcal{S}_{j}\right)
$$

where $m_{\text {in }}\left(\mathcal{S}_{j}\right)$ and $m_{\text {out }}\left(\mathcal{S}_{j}\right)$ are the estimated motion magnitude in the first and last frame of the superframe. We compute $m_{i n}\left(\mathcal{S}_{j}\right)$ and $m_{\text {out }}\left(\mathcal{S}_{j}\right)$ by taking the mean magnitude of the translation, which we estimate by tracking points in the video using KLT. This cost is lower for superframes that have their boundaries aligned with frames containing little or no motion.

The length prior $P_{l}$ is learnt by fitting a log-normal distribution to a histogram of segment lengths of the human created summary selections (cf. Sec. 6). In Fig. 3 we show the prior learnt on the complete dataset. The prior serves as a regularization of the superframes, similar to the shape term in [2].

We optimize the energy of Eq. (1) locally by hill-climbing optimization. First, the superframes are initialized evenly distributed over the video/shot, using the segment length $\left|\mathcal{S}_{j}\right|=\arg \max _{l}\left(P_{l}\right)$. Then,

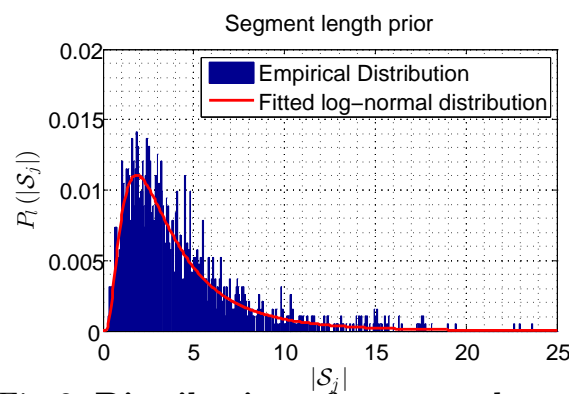

Fig. 3: Distribution of segment lengths. We show the distribution of segment lengths as selected by the study subjects and the fitted log-normal distribution. On the whole dataset we find $\arg \max _{l}\left(P_{l}\right)=1.85$. we iteratively update the boundaries between two superframes to optimize Eq. (1), which leads to segments that have their boundaries aligned to positions suitable for a cut. This optimization is done in a coarse to fine manner, where a boundary movement by $\delta$ frames is proposed. The movement is accepted, if it increases the mean score of Eq. (1), of the two affected superframes. We start from an initial $\delta$ and iteratively update until the algorithm converges. Then, $\delta$ is decreased by one frame and the optimization is re-executed. Fig. 2 illustrates this process. As this optimization is local, only a few iterations are needed until it converges (typically less than 10).

\section{Per-frame Interestingness}

We compute an interestingness score $i_{k}$ for each frame $v_{k}$, as a weighted sum of features that have been found to be related to interestingness $[11,18]$. Thereby we combine low-level information, such as the aesthetic quality (contrast, etc.) and spatio-temperal saliency, with high-level features such as, motion classification and person and landmark detection.

Attention. We use the approach of [5] to predict a human attention score based on spatial [12] and temporal saliency (temporal gradients). As [5] we combine the scores with a non-linear fusion scheme and take the attention score as a single feature in our approach. 
Aesthetics/Quality. To predict the aesthetic quality of a frame we compute colorfulness [3], contrast [14] and the distribution of edges [14].

Presence of landmarks. Landmarks are often of high interest [11]. This is particularly true in holiday videos and has already been exploited in [20] to predict the interestingness of video frames. We follow this idea and use the framework of [8] to classify the scene in a frame as famous or non-famous, based on the presence of famous buildings.

Faces/Persons. As previous works [26, 18], we detect prominent faces or persons in a frame and use them as features for summarization. We detect faces using the algorithm of [27] and persons using [6]. Given a detection, we take the relative area of the bounding box w.r.t. to the frame size as a feature score.

Follow object. Similar to [18] we observe that moving cameras contain implicit information on an objects/events interestingness. A typical pattern in user videos is that an object of interest is tracked by the camera (the movie makers keep it roughly in the center of the video). In order to classify such a motion pattern, we build on recent work in motion segmentation. We separate a set of sparse motion tracks into segments using [4]. Thereby the number of motion segments is automatically determined. From a given segmentation, we find the foreground segment by assuming that it is approximately centered in the frame and spatially compact. Specifically, we take $\arg \min _{\mathbf{O}} \sum\left\|\left(\mu_{\mathbf{o}_{\mathbf{i}}}-\mathbf{c}\right) \sigma_{\mathbf{o}_{\mathbf{i}}}\right\|$ as the foreground segment, where $\mathbf{O}$ is the set of motion segments and the sum is taken over the set of frames a segment is visible. $\mu_{\mathbf{o}_{\mathbf{i}}}$ and $\sigma_{\mathbf{o}_{\mathbf{i}}}$ are the mean and standard deviation of the $\mathrm{x}$ - and y-coordinates of the points in segment $o_{i}$ and $\mathbf{c}$ is the center point of the frame. The background is taken to be the largest remaining motion segment. Given this separation, we estimate a translational motion model for foreground and background. We annotated a set of videos containing 32 follow object motion patterns and computed background and foreground motion magnitude $m_{b}, m_{f}$. From these, we use kernel density estimation over vectors $\mathbf{x}=$ $\left[m_{b}, m_{b}-m_{f}\right]$ in order to estimate $P(\mathbf{x} \mid \mathbf{y}=$ follow object $), P(\mathbf{y}=$ follow object $)$ and $P(\mathbf{x})$. Using Bayes theorem we compute then $P(\mathbf{y}=$ follow object $\mid \mathbf{x})$ and use this probability as a feature.

Combination of features. We combine the above features with a linear model, where we regress the weights $\mathbf{w}$. A frame $v_{k}$ has an interestingness score $i_{k}$

$$
i_{k}=w_{0}+\sum_{i=1}^{N} w_{i} \cdot u_{i}+\sum_{i=1}^{N} \sum_{j=i+1}^{N} w_{i, j} \cdot u_{i} u_{j},
$$

where $u_{i}$ is the score of feature $i$. We use unary and pairwise terms as [18], since such a model is able to capture interactions between features, while it remains sufficiently simple to avoid overfitting and allows for fast training.

We estimate $\mathbf{w}$ using least-squares and the annotated dataset from Sec. 6 . As training ground truth we use a score computed by taking the fraction of selections over views for each frame (examples of such scores are shown in of Fig. 7 as 'human selection'). We randomly sample 100 frames from each training 
video and concatenate them. This way, all videos have the same importance in the learning process. As we randomly sample frames, we repeat this process 50 times and average the resulting weight vectors.

The interestingness score of a superframe $S_{i}$ is simply a sum over the interestingness of its frames:

$$
I\left(S_{i}\right)=\sum_{k=n}^{m} i_{k},
$$

where $n$ and $m$ are start and end frame of superframe $S_{i}$. We also tried other scoring methods, such as taking the maximum or including cluster size, but found this simple sum to work best.

\section{Selecting an Optimal Summary}

Given the set of superframes $\mathcal{S}$, we want to find a subset with a length below a specified maximum $L_{s}$, such that the sum of the interestingness scores is maximized. Formally, we want to solve the following optimization problem:

$$
\begin{aligned}
& \underset{\mathbf{x}}{\operatorname{maximize}} \sum_{i=1}^{n} x_{i} I\left(S_{i}\right) \\
& \text { subject to } \sum_{i=1}^{n} x_{i}\left|S_{i}\right| \leq L_{s},
\end{aligned}
$$

where $x_{i} \in\{0,1\}$ and $x_{i}=1$ indicates that a superframe is selected. Under the assumption of independence between the scores $I\left(S_{i}\right)$, this maximization is a standard 0/1-knapsack problem, where $I\left(S_{i}\right)$ is the value of an item and its length $\left|S_{i}\right|$ its weight. This problem can be solved globally optimal with dynamic programming in pseudo-polynomial time $\mathcal{O}\left(n L_{s}\right)[10]$, with $n=|\mathcal{S}|$.

In this optimization, we do not explicitly account for the possibility that superframes contain redundant information. We also ran experiments where we clustered the superframes beforehand and used an uncorrelated subset of superframes in the optimization to explicitly enforce diversity for the final summary. This however led to no significant improvement, suggesting that study participants choose interesting over representative parts. Furthermore user videos rarely contain multiple interesting, but redundant events (i.e. from our experience it is not necessary to explicitly filter out duplicates).

\section{The SumMe Benchmark}

We introduce a benchmark that allows for the automatic evaluation of video summarization methods. Previous approaches generated video summaries and then let humans assess their quality, in one of the following ways:

i) Based on a set of predefined criteria [25]. The criteria may range from counting the inclusion of predefined important content, the degree of redundancy, summary duration, etc. 
Table 1: The videos in the SumMe dataset. We show consistency and the distribution of segment lengths for each video. The analysis of the consistency (Sec. 6.2) shows that there are certain individual differences but humans still generally agree on what parts of a video are interesting.

\begin{tabular}{|c|c|c|c|c|c|c|c|c|}
\hline \multirow[b]{2}{*}{ Name } & \multirow[b]{2}{*}{ Camera } & \multirow{2}{*}{ Length } & \multirow{2}{*}{$\begin{array}{l}\text { \# of } \\
\text { subj. }\end{array}$} & \multirow{2}{*}{\begin{tabular}{|l|} 
Summary \\
length [\%]
\end{tabular}} & \multicolumn{2}{|c|}{ Segments } & \multicolumn{2}{|c|}{ human consistency } \\
\hline & & & & & avg. \# & avg. length & f-measure & Cronb. $\alpha$ \\
\hline Base jumping & egocentric & $2 \mathrm{~m} 39 \mathrm{~s}$ & 18 & $13.8 \pm 2.0$ & $5.7 \pm 2.2$ & $4.5 \mathrm{~s}$ & 0.26 & 0.77 \\
\hline Bike Polo & egocentric & $1 \mathrm{~m} 43 \mathrm{~s}$ & 15 & $12.3 \pm 3.4$ & $3.9 \pm 1.4$ & $3.8 \mathrm{~s}$ & 0.32 & 0.83 \\
\hline Scuba & egocentric & $1 \mathrm{~m} 14 \mathrm{~s}$ & 17 & $13.2 \pm 2.0$ & $3.5 \pm 1.3$ & $3.4 \mathrm{~s}$ & 0.22 & 0.70 \\
\hline Valparaiso Downhill & egocentric & $2 \mathrm{~m} 53 \mathrm{~s}$ & 15 & $13.6 \pm 1.9$ & $7.7 \pm 4.0$ & $4.2 \mathrm{~s}$ & 0.27 & 0.80 \\
\hline Bearpark climbing & moving & $2 \mathrm{~m} 14 \mathrm{~s}$ & 15 & $14.4 \pm 1.0$ & $5.1 \pm 2.2$ & $4.7 \mathrm{~s}$ & 0.21 & 0.61 \\
\hline Bus in Rock Tunnel & moving & $2 \mathrm{~m} 51 \mathrm{~s}$ & 15 & $12.8 \pm 3.3$ & $5.7 \pm 2.7$ & $4.7 \mathrm{~s}$ & 0.20 & 0.57 \\
\hline Car railcrossing & moving & $2 \mathrm{~m} 49 \mathrm{~s}$ & 16 & $13.2 \pm 2.0$ & $4.9 \pm 2.0$ & $5.4 \mathrm{~s}$ & 0.36 & 0.78 \\
\hline Cockpit Landing & moving & $5 \mathrm{~m} 2 \mathrm{~s}$ & 15 & $12.8 \pm 2.6$ & $7.3 \pm 2.9$ & $6.7 \mathrm{~s}$ & 0.28 & 0.84 \\
\hline Cooking & moving & $1 \mathrm{~m} 27 \mathrm{~s}$ & 17 & $13.8 \pm 1.3$ & $3.2 \pm 1.1$ & $4.3 \mathrm{~s}$ & 0.38 & 0.91 \\
\hline Eiffel Tower & moving & $3 \mathrm{~m} 20 \mathrm{~s}$ & 15 & $11.8 \pm 2.9$ & $5.5 \pm 2.3$ & $4.6 \mathrm{~s}$ & 0.31 & 0.80 \\
\hline Excavators river cross. & moving & $6 \mathrm{~m} 29 \mathrm{~s}$ & 15 & $14.0 \pm 1.2$ & $9.9 \pm 4.7$ & $6.9 \mathrm{~s}$ & 0.30 & 0.63 \\
\hline Jumps & moving & $0 \mathrm{~m} 39 \mathrm{~s}$ & 15 & $14.4 \pm 1.0$ & $2.9 \pm 1.1$ & $2.4 \mathrm{~s}$ & 0.48 & 0.87 \\
\hline Kids playing in leaves & moving & $1 \mathrm{~m} 46 \mathrm{~s}$ & 15 & $13.2 \pm 2.4$ & $4.2 \pm 2.5$ & $4.6 \mathrm{~s}$ & 0.29 & 0.59 \\
\hline Playing on water slide & moving & $1 \mathrm{~m} 42 \mathrm{~s}$ & 15 & $12.6 \pm 2.8$ & $5.2 \pm 3.2$ & $3.2 \mathrm{~s}$ & 0.20 & 0.56 \\
\hline Saving dolphines & moving & $3 \mathrm{~m} 43 \mathrm{~s}$ & 15 & $13.9 \pm 1.3$ & $6.9 \pm 2.9$ & $6.6 \mathrm{~s}$ & 0.19 & 0.21 \\
\hline St Maarten Landing & moving & $1 \mathrm{~m} 10 \mathrm{~s}$ & 17 & $13.9 \pm 1.1$ & $2.8 \pm 1.6$ & $4.8 \mathrm{~s}$ & 0.50 & 0.94 \\
\hline Statue of Liberty & moving & $2 \mathrm{~m} 36 \mathrm{~s}$ & 17 & $10.7 \pm 3.5$ & $3.1 \pm 2.4$ & $7.5 \mathrm{~s}$ & 0.18 & 0.56 \\
\hline Uncut Evening Flight & moving & $5 \mathrm{~m} 23 \mathrm{~s}$ & 15 & $12.1 \pm 2.5$ & $6.3 \pm 3.1$ & $7.6 \mathrm{~s}$ & 0.35 & 0.85 \\
\hline paluma jump & moving & $1 \mathrm{~m} 26 \mathrm{~s}$ & 15 & $12.9 \pm 1.9$ & $3.1 \pm 1.2$ & $4.6 \mathrm{~s}$ & 0.51 & 0.91 \\
\hline playing ball & moving & $1 \mathrm{~m} 44 \mathrm{~s}$ & 16 & $13.9 \pm 1.7$ & $4.7 \pm 2.5$ & $4.3 \mathrm{~s}$ & 0.27 & 0.68 \\
\hline Notre Dame & moving & $3 \mathrm{~m} 12 \mathrm{~s}$ & 15 & $12.9 \pm 2.0$ & $7.6 \pm 3.8$ & $4.1 \mathrm{~s}$ & 0.23 & 0.63 \\
\hline Air Force One & static & $2 \mathrm{~m} 60 \mathrm{~s}$ & 15 & $14.0 \pm 1.5$ & $5.3 \pm 3.0$ & $6.2 \mathrm{~s}$ & 0.33 & 0.85 \\
\hline Fire Domino & static & $0 \mathrm{~m} 55 \mathrm{~s}$ & 15 & $14.0 \pm 1.7$ & $4.0 \pm 2.0$ & $2.2 \mathrm{~s}$ & 0.39 & 0.85 \\
\hline car over camera & static (mostly) & $2 \mathrm{~m} 26 \mathrm{~s}$ & 15 & $12.4 \pm 2.5$ & $4.7 \pm 2.7$ & $5.0 \mathrm{~s}$ & 0.35 & 0.84 \\
\hline Paintball & static (mostly) & $4 \mathrm{~m} 16 \mathrm{~s}$ & 17 & $11.5 \pm 3.3$ & $5.2 \pm 2.2$ & $6.6 \mathrm{~s}$ & 0.40 & 0.87 \\
\hline Mean & & $2 \mathrm{~m} 40 \mathrm{~s}$ & 16 & $13.1 \pm 2.4$ & $5.1 \pm 3.0$ & $4.9 \mathrm{~s}$ & 0.31 & 0.74 \\
\hline
\end{tabular}

ii) Humans are shown two different summaries and are asked to select the better one $[18,22]$. Typically, the summaries are compared to some baseline such as uniform sampling or k-means clustering.

These evaluation methods are problematic, as they are expensive and time consuming as they rely on human judges for each evaluation. The evaluation of the method of [22], for example, required one full week of human labor. Both approaches are discriminative, i.e. they help to tell which summary is better than another, but fail to show what a good summary should look like.

Rather than using the above approaches, we let a set of study subjects generate their own summaries. This was done in a controlled psychological experiment, as described in the next section. We collected multiple summaries for each videos, as there is no true answer for a correct summarization, but rather multiple possible ways. With these human summaries, referred to as human selections, we can compare any method that creates an automatic summary in a repeatable and efficient way. Such automatic vs. human comparison has already been used successfully for keyframes [1,15]. Khosla et al. [15] showed that comparing automatic keyframe summaries to human keyframe selections yields ratings comparable to letting humans directly judge the automatic summaries.

\subsection{Setup}

The SumMe dataset consists of 25 videos covering holidays, events and sports. They are raw or minimally edited user videos, i.e. they have a high compressibility compared to already edited videos. The length of the videos ranges from about 1 to 6 minutes (Overview in Tab. 1). 


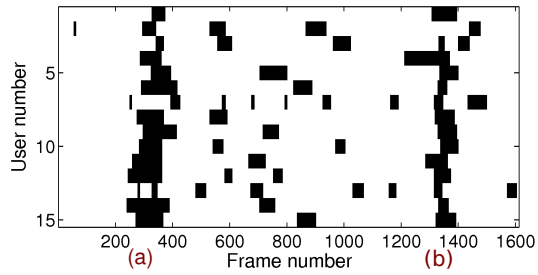

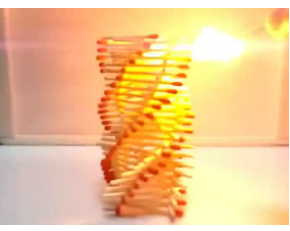

(a) Catching fire

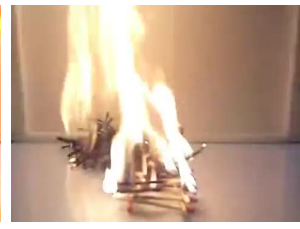

(b) The tower falls

Fig. 4: Consistency of human selections. We show the human selections for the video "Fire Domino", a typical video from our dataset. The selection of a frame is marked in black. As one can see, there is a high consistency among the study participants. They consistently selected the two main events (a) and (b) of the video.

Given a video, the study subjects were asked to produce a summary that contains most of its important content, i.e. that best summarizes the input video. They could use a simple interface that allows to watch, cut and edit a video. We required the summary length $L_{s}$ to be $5 \% \leq L_{s} \leq 15 \%$ to ensure that the input video is indeed summarized rather than being slightly shortened. The videos were shown in random order and the audio track was not included to ensure that the subjects chose based on visual stimuli. A total of 19 male and 22 female subjects, with varying educational background, participated in the study. Ages were ranging from 19 to 39 and all had normal or corrected vision. Each video was summarized by 15 to 18 different people. The total user time of the study amounts to over 40 hours.

An example from our dataset is shown in Fig. 4. The complete experimental data including verbatim instructions, user interface and the human selections can be found in the supplementary material.

\subsection{Human consistency}

In this section we analyze the human selection results in terms of the consistency among the participants.

To assert the consistency of human selections, we propose the use of the pairwise f-measure between them. We will use same consistency measure to evaluate the performance of automatic summaries in Sec. 7. For a human selection $i$, it is defined as follows:

$$
\bar{F}_{i}=\frac{1}{N-1} \sum_{j=1, j \neq i}^{N} 2 \frac{p_{i j} r_{i j}}{p_{i j}+r_{i j}}
$$

where $N$ is the number of human subjects, $p_{i j}$ is the precision and $r_{i j}$ the recall of human selection $i$ using selection $j$ as ground truth. We compute recall and precision on a per-frame basis. This procedure of averaging pairwise comparisons accounts for individual differences in the way humans select segments and was also successfully used in the Berkeley Segmentation Dataset [23]. The dataset has a mean of $\bar{F}=0.31$ (min. 0.18, max. 0.51). Additionally we computed 
the Cronbach alpha, which is a standard measure to assess the reliability of a psychometric test. It is defined as $\alpha=\frac{N \bar{r}}{1+(N-1) \bar{r}}$, where $\bar{r}$ is the mean pairwise correlation between all human selections. The dataset has a mean of $\alpha=0.74$ (min. 0.21, max. 0.94). Ideally $\alpha$ is around 0.9 , while $\alpha>0.7$ is the minimum for a good test $[17$, p. 11, 13].

To summarize, we showed that the most of the videos have a good consistency and it is thus appropriate to train and evaluate computational models on them. This is particularly true, since we use pairwise scores rather than one single reference summary. Generally, we observe the consistency depends on the diversity within a video. Videos that do not have a set of clearly separable events have lower consistency than videos with a set of visually and semantically dissimilar events.

\section{Experiments}

We evaluate our method using the new benchmark and the f-measure defined in Eq. (6). We compare our method to a random, uniform and clustering baseline, as well as a recent method based on visual attention [5]. Further, we compare to the individual human summaries. Ideally, a computer generated summary is as consistent as the best human summary selection. In addition, we also investigate the influence on the performance of the main steps in our pipeline. The results described here are summarized in Tab. 2.

Implementation details. We kept all parameters fixed for all results. When estimating $P_{l}$ and $\mathbf{w}$ we used leave-one training. In the superframe segmentation, we set the initial delta $\delta=0.25 \mathrm{~s}$ and the $\gamma=1$ for all videos. For the interestingness estimation, we computed all image features sparsely every 5 th frame, but processed all frames for motion features. We normalized the feature scores per video to zero mean and unit variance. For the Follow object feature, we used a Gaussian kernel with a window size $h=10$ in the kernel density estimation.

\subsection{Dataset scores}

We characterize the dataset by computing random scores and the upper bound (Tab. 2). The upper bound is defined as the highest reachable score for this dataset, given the human selection and the pairwise f-measure. It would only be 1.0 , if all humans summary selection would be exactly the same.

Additionally, we measure the "human performance", which is the average f-measure of one humans to all the others. We show the worst, average and best scores of the human selections in Tab. 2. The "worst human" score is computed using the summary which is the least similar to the rest of the summaries. The more similar a human selection is to all the others, the higher the score. The best human score is the mean f-measure of the most similar summary w.r.t. all the others, i.e. it mostly contains parts that were selected by many humans. 
Table 2: Quantitative results. We show f-measures at $15 \%$ summary length for our approach, the baselines and the human selections. We highlight the best and second best computational method. Our method consistently shows a high performance scoring higher than the worst human per video.

\begin{tabular}{|c|c|c|c|c|c|c|c|c|c|c|}
\hline & & \multicolumn{2}{|c|}{ Dataset } & \multicolumn{3}{|c|}{ Humans } & \multicolumn{4}{|c|}{ Computational methods } \\
\hline & Videoname & Random & Upper bound & Worst & Mean & Best & Uniform & Cluster. & Att.[5] & Ours \\
\hline \multirow{4}{*}{$\begin{array}{l}80 \\
0\end{array}$} & Base jumping & 0.144 & 0.398 & 0.113 & 0.257 & 0.396 & 0.168 & 0.109 & 0.194 & 0.121 \\
\hline & Bike Polo & 0.134 & 0.503 & 0.190 & 0.322 & 0.436 & $\overline{0.058}$ & $\underline{0.130}$ & 0.076 & 0.356 \\
\hline & Scuba & 0.138 & 0.387 & 0.109 & 0.217 & 0.302 & 0.162 & $\overline{0.135}$ & 0.200 & $\underline{0.184}$ \\
\hline & Valparaiso Downhill & 0.142 & 0.427 & 0.148 & 0.272 & 0.400 & 0.154 & 0.154 & $\underline{0.231}$ & $\overline{0.242}$ \\
\hline \multirow{17}{*}{ 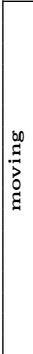 } & Bearpark climbing & 0.147 & 0.330 & 0.129 & 0.208 & 0.267 & 0.152 & 0.158 & 0.227 & 0.118 \\
\hline & Bus in Rock Tunnel & 0.135 & 0.359 & 0.126 & 0.198 & 0.270 & $\underline{0.124}$ & $\overline{0.102}$ & 0.112 & 0.135 \\
\hline & Car railcrossing & 0.140 & 0.515 & 0.245 & 0.357 & 0.454 & $\overline{0.146}$ & $\underline{0.146}$ & 0.064 & 0.362 \\
\hline & Cockpit Landing & 0.136 & 0.443 & 0.110 & 0.279 & 0.366 & 0.129 & $\overline{0.156}$ & 0.116 & 0.172 \\
\hline & Cooking & 0.145 & 0.528 & 0.273 & 0.379 & 0.496 & $\underline{0.171}$ & $\overline{0.139}$ & 0.118 & 0.321 \\
\hline & Eiffel Tower & 0.130 & 0.467 & 0.233 & 0.312 & 0.426 & $\overline{0.166}$ & $\underline{0.179}$ & 0.136 & 0.295 \\
\hline & Excavators river crossing & 0.144 & 0.411 & 0.108 & 0.303 & 0.397 & 0.131 & $\overline{0.163}$ & 0.041 & 0.189 \\
\hline & Jumps & 0.149 & 0.611 & 0.214 & 0.483 & 0.569 & 0.052 & $\overline{0.298}$ & 0.243 & 0.427 \\
\hline & Kids playing in leaves & 0.139 & 0.394 & 0.141 & 0.289 & 0.416 & 0.209 & $\underline{0.165}$ & 0.084 & 0.089 \\
\hline & Playing on water slide & 0.134 & 0.340 & 0.139 & 0.195 & 0.284 & $\underline{0.186}$ & $\overline{0.141}$ & 0.124 & 0.200 \\
\hline & Saving dolphines & 0.144 & 0.313 & 0.095 & 0.188 & 0.242 & $\underline{0.165}$ & 0.214 & 0.154 & 0.145 \\
\hline & St Maarten Landing & 0.143 & 0.624 & 0.365 & 0.496 & 0.606 & $\overline{0.092}$ & 0.096 & 0.419 & $\underline{0.313}$ \\
\hline & Statue of Liberty & 0.122 & 0.332 & 0.096 & 0.184 & 0.280 & $\underline{0.143}$ & 0.125 & 0.083 & $\overline{0.192}$ \\
\hline & Uncut Evening Flight & 0.131 & 0.506 & 0.206 & 0.350 & 0.421 & $\overline{0.122}$ & 0.098 & 0.299 & $\underline{0.271}$ \\
\hline & paluma jump & 0.139 & 0.662 & 0.346 & 0.509 & 0.642 & $\underline{0.132}$ & 0.072 & 0.028 & $\overline{0.181}$ \\
\hline & playing ball & 0.145 & 0.403 & 0.190 & 0.271 & 0.364 & $\overline{0.179}$ & $\underline{0.176}$ & 0.140 & 0.174 \\
\hline & Notre Dame & 0.137 & 0.360 & 0.179 & 0.231 & 0.287 & 0.124 & 0.141 & 0.138 & 0.235 \\
\hline \multirow{7}{*}{ 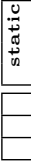 } & Air Force One & 0.144 & 0.490 & 0.185 & 0.332 & 0.457 & 0.161 & 0.143 & $\underline{0.215}$ & 0.318 \\
\hline & Fire Domino & 0.145 & 0.514 & 0.170 & 0.394 & 0.517 & 0.233 & 0.349 & $\overline{0.252}$ & 0.130 \\
\hline & car over camera & 0.134 & 0.490 & 0.214 & 0.346 & 0.418 & 0.099 & $\underline{0.296}$ & $\overline{0.201}$ & 0.372 \\
\hline & Paintball & 0.127 & 0.550 & 0.145 & 0.399 & 0.503 & 0.109 & $\overline{0.198}$ & $\underline{0.281}$ & 0.320 \\
\hline & mean & 0.139 & 0.454 & 0.179 & 0.311 & 0.409 & 0.143 & 0.163 & 0.167 & 0.234 \\
\hline & relative to upper bound & $31 \%$ & $100 \%$ & $39 \%$ & $68 \%$ & $90 \%$ & $31 \%$ & $36 \%$ & $37 \%$ & $52 \%$ \\
\hline & relative to average human & $45 \%$ & $146 \%$ & $58 \%$ & $100 \%$ & $131 \%$ & $46 \%$ & $53 \%$ & $54 \%$ & $75 \%$ \\
\hline
\end{tabular}

\subsection{Baselines}

We compare our approach to the following baselines:

Uniform sampling. We uniformly select $\mathrm{K}$ segments of length $\arg \max _{l}\left(P_{l}\right)$, such that the final summary length is $\leq L_{s}$ (15\% of the input).

Clustering. We computed color histograms with $16^{3}$ dimensions for each frame and averaged these per superframe. Then, we clustered the superframes with [7], using the affinity of [18]. Given this clustering, we use the cluster centers as candidates for the final summary and select a subset using Eq. (5).

Visual Attention. Recently [5] proposed an approach for keyframe selection based on the principles of human attention ( $c f$. Sec. 4). As this method produces keyframes, we selected $\mathrm{K}$ segments of length $\arg \max _{l}\left(P_{l}\right)$ around the highest scored frames, such that the final summary is of length $L_{s}$ (15\% of the input video).

\section{$7.3 \quad$ Results}

As can be see from Tab. 2, our method outperforms all baselines. Our method has an average performance of $52 \%$, while the strongest baseline reaches $37 \%$, relative to the upper bound. If we compare to the human consistency (the human 'performance'), we can see that our method even outperforms, on average, the worst human of each video. Furthermore it reaches a performance comparable to the average human summary in many cases. Our method is able to find the 
important segments of a video and to produce an informative summary from them. The proposed features capture the central aspects of a video.

The highest average performance is achieved on static cameras. This is not surprising as in such a setting simple features are often sufficient to find an event of interest (e.g. temporal gradients). While our method performs well in all settings (static, moving and egocentric), it has a low performance for certain videos, e.g. the video "Base jumping". This video contains fast motion and subtle semantics that define important events of the video, such as opening the parachute or the landing. These are difficult to capture based on the used image and motion features, which leads to a low performance for this video.

In Fig. 5 we show the quantitative performance over the whole video for different summarization ratios and a visualization of a few automatic summaries in Fig. 7. We refer the reader to the supplementary material for quantitative and especially qualitative results on all videos.

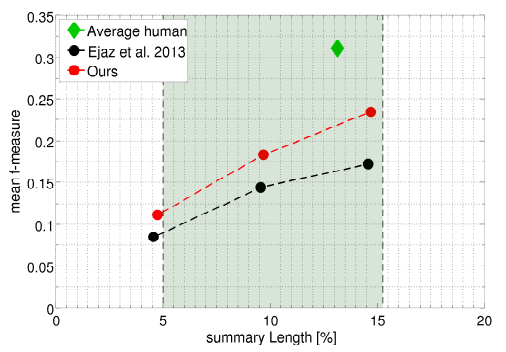

Fig. 5: Quantitative results. We compare our method (red) to the visual attention [5] baseline (black) and the average human performance (green) over the entire dataset. Automatic summaries are computed for lengths $\leq 5 \%, 10 \%$ and $15 \%$.

\subsection{Performance of the individual components}

Interestingness. We investigate the importance and reliability of the individual interestingness features. In Fig. 6a we show the performance gain by adding a feature to the set of used features (the difference in performance of (not) using a feature). As could be expected, general features perform best, as they can potentially help on all videos. Somewhat surprisingly, a feature as simple as colorfulness leads to a high performance gain. Additionally, we observe a large improvement by using the detection of landmarks and a camera that follows a moving object. This is despite the fact, that only a fraction of videos contains either of these. However, if e.g. a landmark appears in the video, this is a strong indicator that such a part should be selected for a summary. When combining the individual features, they can predict what parts of a video should be selected for the automatic summary (see performance in Tab. 6b). However, the features cannot capture what is interesting in all cases. Lacking a temporal smoothing the scores are often noisy and, when selecting frames based on this score, create disruptive segments. We target these problems by temporal smoothing, as we discuss in the following.

Superframes. We analyze the performance gain by using temporal segmentation (Tab. 6b). Instead of using per-frame interestingness scores, we compute a score per temporal window. We compare the proposed superframes to segments 


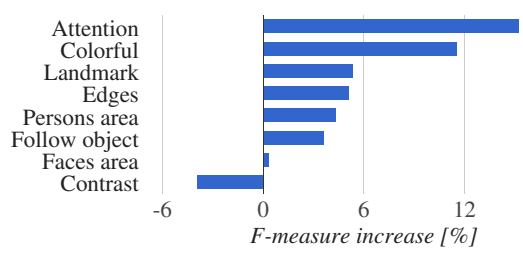

Feature performance. We show the increase in performance by adding a feature to the set of used features.

\begin{tabular}{|c|l|l|}
\hline & \multicolumn{2}{|c|}{ mean f-measure } \\
\hline Segmentation & per frame & per segment \\
\hline \hline Single frame & $0.217(70 \%)$ & $0.035(10 \%)$ \\
Fixed length & $0.222(72 \%)$ & $0.151(44 \%)$ \\
Clustering & $0.228(73 \%)$ & $0.155(45 \%)$ \\
Superframes & $0.234(75 \%)$ & $0.170(49 \%)$ \\
\hline
\end{tabular}

Temporal segmentation: We report the performance of different temporal segmentations. Percent scores are relative to the average human.

Fig. 6: Weights and performance of the individual steps in the pipeline (See text).

of fixed length, where we set the length to the optimal value according to the length prior $P_{l}$. As an additional baseline we use clustering to select keyframes (k-means) and use these as centers to create segments, which partition the video into shots (with segment boundaries in the middle between two keyframes). Each segment is scored according to Eq. (4) and the summary is optimized using Eq. (5), such that the final summary length maximally $15 \%$ of the initial video. As we want to analyze the quality of the created segments, we report fmeasure on the per-frame and also on the segment level. To compute recall and precision of segments, we compute the intersection over union of the segments and threshold it at 0.25 .

As expected, smoothing over a temporal neighborhood leads to an increased performance, especially on the segment level. While the main aim of the superframes is to produce aesthetically pleasing summaries, we can observe that using these motion aligned segments yields a better performance. This indicates that using such a grouping is indeed more semantically logical. For qualitative differences in the produced summaries, we refer the reader to the videos in the supplementary material or on our website.

\section{Conclusion}

In this work we proposed a novel temporal superframe segmentation for user videos and a method to produce informative summaries from them. To score the superframes we proposed a set of interestingness features and showed that they capture what is important well. With the use of a 0/1-knapsack formulation, we optimized the interestingness of the final summary, while remaining within a given time budget.

The evaluation of our method shows that it is generally able to create good automatic summaries, often reaching the performance of humans. Nonetheless, video summarization is still in its beginnings. The contribution of our benchmark with multiple human summaries per video makes it possible to gain additional insights into what humans rate as important. This will help develop new features and methods in the future. 


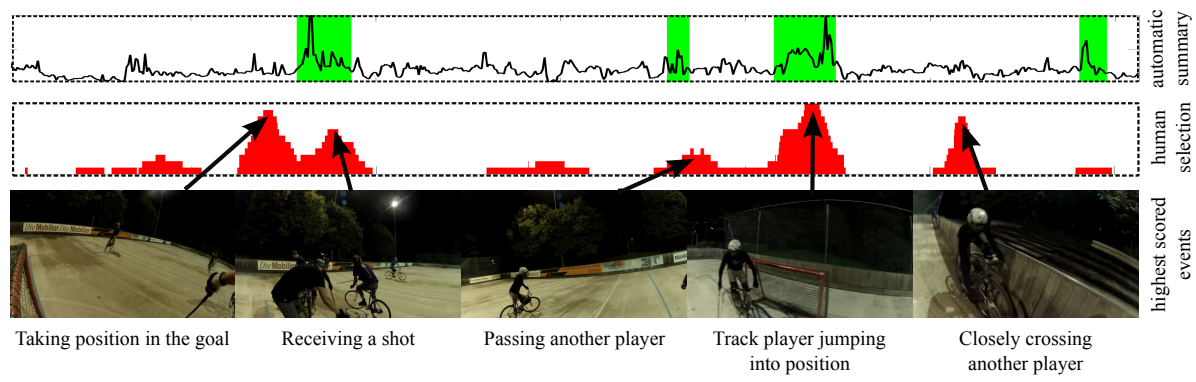

Video "Bike Polo"

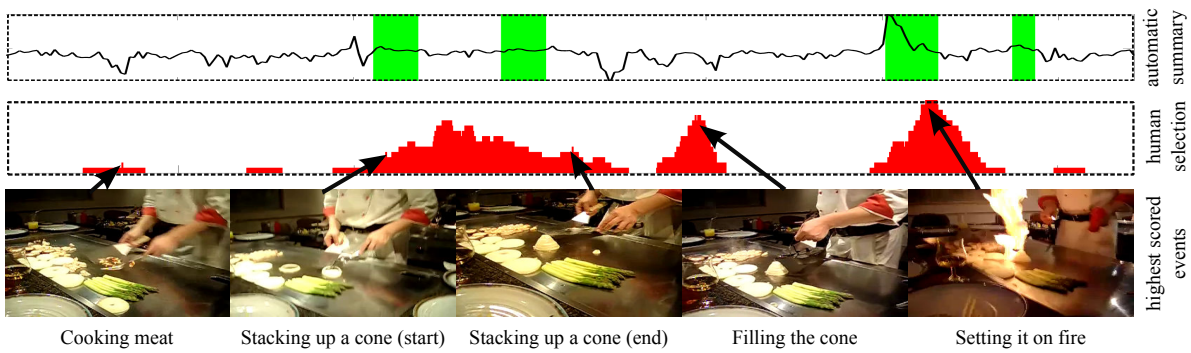

Video "Cooking"

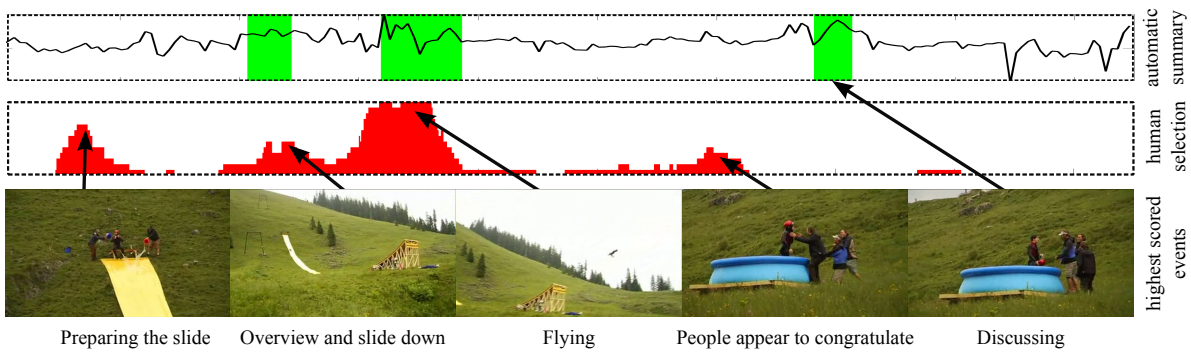

Video "Jumps"

Fig. 7: Example summaries. For each video we show the predicted interestingness score (black) and the selected segments (green) on top. In the middle we show the human scores (red). The human score is computed as the ratio of selections over views, per frame. Peaks in the human score indicate that this part was often selected by humans, while a peak in the interestingness score indicates a high prediction for this part. Our method correctly selects the most important events and produces a compact and interesting summary from them. The superframe segementation ensures that the cuts between the segments are smooth. Best viewed in color. All generated summaries are given in the supplementary material.

Acknowledgements. We thank Michel Druey and the Varcity team for fruitful discussions and help. This work was supported by the European Research Council (ERC) under the project VarCity (\#273940) and the Swiss CTI under project no. 15769.1. 


\section{References}

1. de Avila, S.E.F., Lopes, A.P.B., da Luz Jr., A., de A. Arajo, A.: VSUMM: a mechanism designed to produce static video summaries and a novel evaluation method. Pattern Recognition Letters (2011)

2. Van den Bergh, M., Boix, X., Roig, G., de Capitani, B., Van Gool, L.: SEEDS: superpixels extracted via energy-driven sampling. ECCV (2012)

3. Datta, R., Joshi, D., Li, J., Wang, J.Z.: Studying aesthetics in photographic images using a computational approach. ECCV (2006)

4. Dragon, R., Ostermann, J., Van Gool, L.: Robust Realtime Motion-Split-AndMerge for Motion Segmentation. GCPR (2013)

5. Ejaz, N., Mehmood, I., Wook Baik, S.: Efficient visual attention based framework for extracting key frames from videos. Signal Processing: Image Communication (2013)

6. Felzenszwalb, P.F., Girshick, R.B., McAllester, D., Ramanan, D.: Object detection with discriminatively trained part based models. PAMI (2010)

7. Frey, B.J., Dueck, D.: Clustering by passing messages between data points. Science (2007)

8. Gammeter, S., Bossard, L., Quack, T., Van Gool, L.: I know what you did last summer: object-level auto-annotation of holiday snaps. ICCV (2009)

9. Goldman, D., Curless, B.: Schematic storyboarding for video visualization and editing. ACM Trans. on Graphics (2006)

10. Goodrich, M.T., Tamassia, R.: Algorithm Design: Foundation, Analysis and Internet Examples. John Wiley \& Sons (2006)

11. Gygli, M., Grabner, H., Riemenschneider, H., Nater, F., Van Gool, L.: The interestingness of images. ICCV (2013)

12. Hou, X., Harel, J., Koch, C.: Image signature: Highlighting sparse salient regions. PAMI (2012)

13. Huang, T., Mehrotra, S.: Adaptive key frame extraction using unsupervised clustering. Proc. Image Processing (1998)

14. Ke, Y., Tang, X., Jing, F.: The design of high-level features for photo quality assessment. CVPR (2006)

15. Khosla, A., Hamid, R., Lin, C., Sundaresan, N.: Large-Scale Video Summarization Using Web-Image Priors. CVPR (2013)

16. Kim, G., Sigal, L., Xing, E.P.: Joint Summarization of Large-scale Collections of Web Images and Videos for Storyline Reconstruction. CVPR (2014)

17. Kline, P.: The handbook of psychological testing. Psychology Press (2000)

18. Lee, Y.J., Ghosh, J., Grauman, K.: Discovering important people and objects for egocentric video summarization. CVPR (2012)

19. Liu, D., Hua, G., Chen, T.: A hierarchical visual model for video object summarization. PAMI (2010)

20. Liu, F., Niu, Y., Gleicher, M.: Using Web Photos for Measuring Video Frame Interestingness. IJCAI (2009)

21. Liu, Z., Zavesky, E., Shahraray, B.: Brief and high-interest video summary generation: evaluating the AT\&T labs rushes summarizations. ACM WS on Video summarization (2008)

22. Lu, Z., Grauman, K.: Story-Driven Summarization for Egocentric Video. CVPR (2013)

23. Martin, D., Fowlkes, C., Tal, D., Malik, J.: A database of human segmented natural images and its application to evaluating segmentation algorithms and measuring ecological statistics. ICCV (2001) 
24. Mascelli, J.V.: The five C's of cinematography. Cine/Grafic Publications (1965)

25. Over, P., Smeaton, A.F., Awad, G.: The TRECVID 2008 BBC rushes summarization evaluation. Proc. ACM WS on Video summarization (2008)

26. Smith, M., Kanade, T.: Video skimming and characterization through the combination of image and language understanding. Proc. on Content-Based Access of Image and Video Database (1998)

27. Viola, P., Jones, M.: Robust real-time face detection. IJCV (2004)

28. Wolf, W.: Key frame selection by motion analysis. Acoustics, Speech, and Signal Processing (1996) 